\title{
CONSTRUCTION OF A FAMILY OF NON-SELF-DUAL GAUGE FIELDS
}

\author{
IGNACIO SOLS
}

\begin{abstract}
Using the generalization of vector bundles by reflexive sheaves recently introduced by $\mathrm{R}$. Hartshorne in [2] we construct a 15-dimensional family of nontrivial complex gauge fields $(U, E, \nabla)$ which are not self-dual nor anti-self-dual. ( $U$ is an affine neighborhood in $Q_{4}=\operatorname{Gr}\left(2, \mathrm{C}^{4}\right.$ ) of the stereographic compactification $S^{4}$ of $\mathbf{R}^{4}, E$ is a vector bundle on $U$ and $\nabla$ is a connection on it whose curvature $\phi$ satisfies the inequalities ${ }^{*} \phi \neq \phi$ and ${ }^{*} \phi \neq-\phi$.)
\end{abstract}

In $[4,5]$ Isenberg et al. and Manin have studied gauge fields whose curvature is neither self-dual nor anti-self-dual. We miss however actual examples of the theory and this we will give in this note. Moreover, we introduce in twistor theory the tool of reflexive sheaves (a kind of vector bundle with singularities) whose interest is very recent in geometry, and they seem to play a roll also in this context. We start by recalling the characterizations of such fields, which are well known by now. We use the language of coherent sheaves [1].

The complexification of the stereographic compactification $S^{4}$ of $\mathbb{R}^{4}$ is the complex 4-dimensional projective smooth quadric $Q_{4}$. This is usually referred to by geometers as the Klein quadric. It can also be viewed as the Grassmann variety parametrizing all lines in projective space $\mathbf{P}^{3}=\mathbf{P}\left(\mathbf{C}^{4}\right)$ and $S^{4}$ as the subset of points corresponding to the lines $L=\mathbf{P}^{1}(V)$ such that $V \cong \mathbf{C}^{2}$ is invariant under the antiholomorphic map $\mathbf{C}^{4} \rightarrow \mathbf{C}^{4}$ consisting of multiplication by $j \in \mathbf{H}$ (quaternions) when we decompose $\mathbf{C}^{4} \cong \mathbf{H} \oplus j \mathbf{H}$.

Our construction depends on 14 complex parameters and produces an affine neighborhood $U$ of $S^{4}$ in $Q_{4}$ and a vector bundle on $U$ together with with a connection on it whose curvature $\phi$ is neither self-dual $\left({ }^{*} \phi \neq \phi\right)$ nor anti-self-dual $\left({ }^{*} \phi \neq-\phi\right)$.

Such a vector bundle is not trivial, in the sense that it is not a direct sum of line bundles, and it does not extend to the whole of $\mathbf{P}^{3} \times \mathbf{P}^{3^{*}}$. We leave open the problem of determining whether the gauge fields we have constructed are currentless, in the sense of satisfying the homogeneous Yang-Mills equation $\nabla^{*} \phi=0$.

Let $\mathbf{P}^{3^{*}}$ be the dual projective space and let $X \subseteq P^{3} \times P^{3^{*}}$ be the variety of pairs $(x, \pi)$ consisting of a point $x$ and a plane $\pi$ such that $x \in \pi$. It can be viewed as the parameter space of the lines of $Q_{4}$ : a point $(x, \pi)$ of $X$ corresponds to the line in $Q_{4}$ consisting of all $l \in Q_{4}$ such that for the associated line $L$ of $P^{3}, x \in L \subseteq \pi$. Let $U$ be an affine open subset of $Q_{4}$ and denote by $X^{\prime}$ the Zariski open subset of $X$ corresponding to those lines of $G$ intersecting $U$. The following has been proved by Isenberg-Yaskin-Green [4] and by Witten [7] (see also [5]): Given a rank 2 vector

Received by the editors March 21, 1983 and, in revised form, August 15, 1985.

1980 Mathematics Subject Classification. Primary 14F05; 81E10. 
bundle $E^{\prime}$ on $X^{\prime}$ with trivial restriction to the quadric surface $X_{l}=\{(x, \pi) \in X \mid$ $x \in L \subseteq \pi\}$, for each $l \in U$, is equivalent to a rank 2 vector bundle on $U$ and a connection on it. More explicitly, if $F \subseteq Q_{4} \times X$ is the flag variety (i.e. the graph of the incidence relation) and

$$
Q_{4} \stackrel{\mathrm{pr}_{1}}{\leftarrow} F \stackrel{\mathrm{pr}_{2}}{\rightarrow} X, \quad U \stackrel{\mathrm{pr}_{1}^{\prime}}{\leftarrow} F^{\prime} \stackrel{\mathrm{pr}_{2}^{\prime}}{\rightarrow} X^{\prime},
$$

the obvious projections and their restrictions, then $\operatorname{pr}_{2}^{\prime *} E^{\prime}$ has a natural connection $\nabla$ defined by taking as $\nabla$-horizontal sections of $\operatorname{pr}_{2}^{\prime *} E^{\prime}$ those sections that are constant along the fibers of $\operatorname{pr}_{2}^{\prime}$. Then $\operatorname{pr}_{1 *}^{\prime} \operatorname{pr}_{2}^{\prime *} E^{\prime}$ is the bundle on $U$ and $\operatorname{pr}_{1 *}^{\prime}$ is the connection on it corresponding to $E^{\prime}$. The curvature of this connection is self-dual or anti-self-dual if and only if $E^{\prime}$ is the pullback of some bundle on $\mathbf{P}^{3}$ or $\mathbf{P}^{3^{*}}$.

For our construction, we think of $Q_{4}$ as embedded in $\mathbf{P}^{5}$ and choose (five parameters) a smooth hyperplane section $Q_{3}$ of $Q_{4}$ disjoint from $S^{4}$. Our open set $U$ will be $Q_{4} \backslash Q_{3}$. For any point $x$ of $\mathbf{P}^{3}$ the plane of $G$ corresponding to all lines of $\mathbf{P}^{3}$ passing through $x$ intersects $Q_{3}$ exactly in one line (for $Q_{3}$ is a quadric threefold and such threefolds do not contain any plane [3]). Denoting this line by $\varphi(x)$ we obtain an inclusion map $\varphi: \mathbf{P}^{3} \rightarrow X$ and we denote by $Y$ its image. As $Y$ is just the subvariety of $X$ corresponding to those lines of $Q_{4}$ contained in $Q_{3}$, the open set $X \backslash Y$ is nothing but $X^{\prime}$ as defined above. Now we use Hartshorne's techniques [2] of reflexive sheaves (i.e. coherent sheaves isomorphic to their double duals) to construct a bundle on it.

First, we observe that all line bundles on $\mathbf{P}^{3} \times \mathbf{P}^{3 *}$ are of the form $\mathcal{O}_{\mathbf{P}^{3} \times \mathbf{P}^{3 *}}(n, m)$ $=p_{1}^{*} \mathcal{O}_{\mathbf{P}^{3}}(n) \otimes p_{2}^{*} \mathcal{O}_{\mathbf{p}^{3 *}}(m)$. In particular, the ideal sheaf defining $X$ in $\mathbf{P}^{3} \times \mathbf{P}^{3 *}$ is $\mathcal{O}_{\mathbf{P}^{3} \times \mathbf{p}^{3 *}(-1,-1)}$ so that we can write an exact sequence

$$
0 \rightarrow \mathcal{O}_{\mathbf{p}^{3} \times \mathbf{p}^{3 *}}(-1,-1) \rightarrow \mathcal{O}_{\mathbf{p}^{3} \times \mathbf{p}^{3 *}} \rightarrow \mathcal{O}_{X} \rightarrow 0 .
$$

We also use the fact that

$$
H^{i}\left(\mathcal{O}_{\mathbf{P}^{3} \times \mathbf{p}^{3 *}}(n, m)\right)=\underset{j+k=i}{\bigoplus} H^{j}\left(\mathcal{O}_{\mathbf{P}^{3}}(n) \otimes H^{k}\left(\mathcal{O}_{\mathbf{P}^{3 *}}(m)\right)\right)
$$

and $H^{i}\left(\mathcal{O}_{\mathbf{P}^{3}}(n)\right)=0$ for $i=1,2$ and any value of $n$. Furthermore, $H^{0}\left(\mathcal{O}_{\mathbf{P}^{3}}(n)\right)=0$ for $n<0$, so by duality $H^{3}\left(\mathcal{O}_{\mathbf{p}^{3}}(n)\right)=0$ for $n \geqslant-3$.

The first and last terms of the sequence

$$
\operatorname{Ext}^{1}\left(\mathcal{O}_{X}, \mathcal{O}_{X}\right) \rightarrow \operatorname{Ext}^{1}\left(\mathscr{I}_{Y}, \mathcal{O}_{X}\right) \rightarrow \operatorname{Ext}^{2}\left(\mathcal{O}_{Y}, \mathcal{O}_{X}\right) \rightarrow \operatorname{Ext}^{2}\left(\mathcal{O}_{X}, \mathcal{O}_{X}\right)
$$

are zero (so that the two in the middle are isomorphic). Indeed, they are isomorphic to $H^{1}\left(\mathcal{O}_{X}\right)$ and $H^{2}\left(\mathcal{O}_{X}\right)$ respectively, so both of them vanish because the first and last terms of the two sequences

$$
\begin{aligned}
& H^{1}\left(\mathcal{O}_{\mathbf{p}^{3} \times \mathbf{p}^{3 *}}\right) \rightarrow H^{1}\left(\mathcal{O}_{X}\right) \rightarrow H^{2}\left(\mathcal{O}_{\mathbf{p}^{3} \times \mathbf{p}^{3 *}}(-1,-1)\right), \\
& H^{2}\left(\mathcal{O}_{\mathbf{p}^{3} \times \mathbf{p}^{3 *}}\right) \rightarrow H^{2}\left(\mathcal{O}_{X}\right) \rightarrow H^{3}\left(\mathcal{O}_{\mathbf{p}^{3} \times \mathbf{p}^{3 *}}(-1,-1)\right)
\end{aligned}
$$

also vanish as a consequence of the above remarks. 
We recall in the next place that there is a spectral sequence relating the "local and global Ext functors," whose second terms are

$$
E_{2}^{p, q}=H^{p}\left(\mathscr{E} x t^{q}\left(\mathcal{O}_{Y}, \mathcal{O}_{X}\right)\right)
$$

converging to $E^{n}=\operatorname{Ext}^{n}\left(\mathcal{O}_{Y}, \mathcal{O}_{X}\right)$. Since $\mathscr{E} x t^{q}\left(\mathcal{O}_{Y}, \mathcal{O}_{X}\right)=0$ for $q \neq \operatorname{codim} Y=2$, this spectral sequence degenerates, and we obtain

$$
\operatorname{Ext}^{2}\left(\mathcal{O}_{Y}, \mathcal{O}_{X}\right)=H^{0}\left(\mathscr{E} x t^{2}\left(\mathcal{O}_{Y}, \mathcal{O}_{X}\right)\right)
$$

Since $\omega_{\mathbf{p}^{3}} \simeq \mathcal{O}_{\mathbf{p}^{3}}(-4)$, we have $\omega_{\mathbf{p}^{3} \times \mathbf{P}^{3 *}} \simeq \mathcal{O}_{\mathbf{p}^{3} \times \mathbf{P}^{3 *}}(-4,-4)$. Using Proposition 8.20, Chapter II of [5], we obtain

$$
\omega_{X} \simeq \omega_{\mathbf{P}^{3} \times \mathbf{P}^{3 *}} \otimes \mathcal{O}_{\mathbf{P}^{3} \times \mathbf{P}^{3 *}}(1,1) \otimes \mathcal{O}_{X} \simeq \mathcal{O}_{X}(-3,-3) .
$$

We now can compute, using the isomorphism $Y \simeq \mathbf{P}^{3}$,

$$
\begin{aligned}
\mathscr{E} x t^{2}\left(\mathcal{O}_{Y}, \mathcal{O}_{X}\right) & \simeq \mathscr{E} x t^{2}\left(\mathcal{O}_{Y}, \mathcal{O}_{X}\right)(-3,-3) \otimes \mathcal{O}_{X}(3,3) \\
& \simeq \omega_{Y} \otimes \mathcal{O}_{X}(3,3) \simeq \mathcal{O}_{Y}(-4) \otimes \mathcal{O}_{X}(3,3) \simeq \mathcal{O}_{Y}(2)
\end{aligned}
$$

(cf. [2, III.6.7]). Summing up, we obtain

$$
\operatorname{Ext}^{1}\left(\mathscr{I}_{Y}, \mathcal{O}_{X}\right) \simeq H^{0}\left(\mathcal{O}_{Y}(2)\right) \text {. }
$$

Every element $\xi$ of this vector space over $\mathbf{C}$ of dimension 10 defines, up to a nonzero scalar, a quadric surface $Z$ in $Y \simeq \mathbf{P}^{3}$ when we see it in $H^{0}\left(\mathcal{O}_{Y}(2)\right)$, and when we see it in $\operatorname{Ext}^{1}\left(\mathscr{I}_{Y}, \mathcal{O}_{X}\right)$ it defines a torsion free coherent sheaf $E$ of rank 2 on $X$ appearing as an extension

$$
0 \rightarrow \mathcal{O}_{X} \rightarrow E \rightarrow \mathscr{I}_{Y} \rightarrow 0
$$

Let $x$ be a point of the scheme $X$. We now consider the sequences of local cohomology:

$$
\begin{aligned}
& H_{\mathfrak{m}_{x}}^{i}\left(\mathcal{O}_{X, x}\right) \rightarrow H_{\mathfrak{m}_{x}}^{i}\left(E_{x}\right) \rightarrow H_{\mathfrak{m}_{x}}^{i}\left(\mathscr{I}_{Y, x}\right), \\
& H_{\mathfrak{m}_{x}}^{i-1}\left(\mathcal{O}_{Y, x}\right) \rightarrow H_{\mathfrak{m}_{x}}^{i}\left(\mathscr{I}_{Y, x}\right) \rightarrow H_{\mathfrak{m}_{x}}^{i}\left(\mathcal{O}_{X, x}\right) .
\end{aligned}
$$

As $Y$ and $X$ are Cohen-Macaulay schemes, they have depth $Y=3$, and depth $X=5$, so that $H^{i}\left(\mathcal{O}_{Y, x}\right)=0$ for $i<3$ and $H^{i}\left(\mathcal{O}_{X, x}\right)=0$ for $i<5$. Therefore $H^{i}\left(\mathscr{I}_{Y, x}\right)=0$ for $i<4$, so that $H^{i}\left(E_{x}\right)=0$ for $i<4$. We can therefore apply Proposition 1 of [6] asserting that, under this hypothesis, $E$ is locally free everywhere but in the points where $\xi$ fails to generate $\mathcal{O}_{Y}(2)$, i.e. in the quadric surface $Z$. We can also assert that $E$ is reflexive, i.e. that $E^{* *} \simeq E$ because this property is characterized in [4, Proposition 1.3] by the fact that depth $\left(E_{x}\right) \geqslant 2$ at every point $x \in X$ such that $\operatorname{dim} \mathcal{O}_{X, x} \geqslant 2$.

As $E$ is locally free at all points of $X^{\prime}=X-Y$, we can see the restriction $E^{\prime}=E \otimes_{\mathcal{O}_{X}} \mathcal{O}_{X^{\prime}}$ of $E$ to $X^{\prime}$ as a bundle on $X^{\prime}$. We observe that this bundle $E^{\prime}$ is not the restriction of any bundle $\mathscr{F}$ on $X$. Indeed, in that case $\mathscr{F}$ and $E$ would be two reflexive sheaves isomorphic outside of a closed subset $Y$ of codimension two and it is an immediate consequence of Proposition 1.6(iii), \$1 of [2] that this implies they are in fact isomorphic. This would contradict that $E$ is not locally free. This observation proves the two facts announced at the introduction: First, the bundle $E^{\prime}$ does not extend (a fortiori) to a bundle on $\mathbf{P}^{3} \times \mathbf{P}^{3 *}$. Second, it is not a direct sum of line bundles on $X^{\prime}$, as such bundles extend in fact to line bundles on $X$. For all 
$l \in U$, the restriction $E_{X_{l}}$ of $E^{\prime}$ to the quadric $X_{l}$ (associated to $l$ further above) is trivial. Indeed, tensoring with $\mathcal{O}_{X}$ the last exact sequence we get

$$
0 \rightarrow \mathcal{O}_{X_{l}} \rightarrow E_{X_{l}} \rightarrow \mathcal{O}_{X_{l}} \rightarrow 0
$$

because $X_{l} \subseteq X^{\prime}$. As $\operatorname{Ext}^{1}\left(\mathcal{O}_{X_{l}}, \mathcal{O}_{X_{l}}\right)=H^{1}\left(\mathcal{O}_{X_{l}}\right)=0$, this sequence splits.

If $E^{\prime}$ were the pullback $p_{1}^{\prime *} V$ of some bundle $V$ on $\mathbf{P}^{3}$ along the natural projection $X^{\prime} \stackrel{p_{1}^{\prime}}{\rightarrow} P^{3}$, then $p_{1}^{*} V$ would be isomorphic to $E$ because two reflexive sheaves that are isomorphic up to codimension 2 are isomorphic. This would contradict the fact $E$ is not locally free. Analogously, $E^{\prime}$ is not a pullback of a bundle in $\mathbf{P}^{3}$. This finishes our construction, which depends on 14 complex parameters ( 5 and 9 for the choice of $Q_{3}$ and $\xi$ ).

We have not been able to determine whether $H^{2}\left(\mathscr{E} n d E^{\prime}(-3,-3)\right)$ is zero or not, which according to Isenberg et al. [4] and Manin [5] is the condition for the encoded connection to satisfy the the Yang-Mills equations.

The bundles we obtain have rank 2, but the same construction produces bundles $E$ of any rank $r \geqslant 2$ by starting with a sequence

$$
0 \rightarrow \mathcal{O}_{X}^{r} \rightarrow E \rightarrow \mathscr{I}_{Y} \rightarrow 0
$$

defined by a point $\xi \in \mathbf{P}\left(\operatorname{Ext}^{1}\left(\mathscr{I}_{Y}, \mathcal{O}_{X}^{r}\right)\right)=\mathbf{P}\left(H^{0}\left(\mathcal{O}_{P^{3}}(2)\right)^{r}\right)$.

We finally note that perhaps reflexive sheaves should be taken into account in the context of twistors. In fact, although it is not true in general that real analytic bundles on $S^{4}$ extend to holomorphic bundles on the whole of $Q_{4}$, those admitting a connection with self-dual curvature extend to a reflexive sheaf on $Q_{4}$. On the other hand, working with reflexive sheaves is similar to working with bundles.

\section{REFERENCES}

1. R. Hartshorne, Algebraic geometry, Graduate Texts in Math., no. 52, Springer-Verlag, New York, 1977.

2. Stable reflexive sheaves, Math. Ann. 254 (1980), 121-176.

3. W. V. D. Hodge and D. Pedoe, Methods of algebriac geometry, Vol. II, Cambridge Univ. Press, New York, 1947.

4. J. Isenberg, P. B. Yaskin, and P. S. Green, Non-self-dual gauge fields, Phys. Lett. B 78 (1978), 462.

5. Yu. I. Manin, Gauge fields and holomorphic geometry, Current Problems in Math., Vol. 17, VINITI, Moscow, 1981, pp. 3-55. (Russian)

6. J.-P. Serre, Sur les modules projectives, Exposé 2, Séminaire Dubreil-Pisot 1960-61, Secrétariat Math., Paris, 1961.

7. E. Witten, An interpretation of classical Yang-Mills theory, Phys. Lett. B 77 (1978), 394-398.

Departmento de algebra, Facultad de Matematicas, Universidad Complutense, Madrid 28040, SPAIN 